\title{
visual neuroscience
}

\section{DETAILED INFORMATION FOR CONTRIBUTORS}

AIMS AND SCOPE. Visual Neuroscience publishes papers based on original experimental or theoretical work concerned with the biological substrates of vision, including the neural mechanisms involved in visually guided behavior and perception. Studies based exclusively on clinical, psychophysical, or behavioral methods will be considered only if they are designed to address a question concerning the neural mechanisms of vision. The journal features full-length Research Articles, Review Articles, as well as Brief Communications.

ORIGINALITY AND COPYRIGHT. To be considered for publication in Visual Neuroscience a manuscript cannot have been published previously, nor can it be under review for publication elsewhere. Papers with multiple authors are reviewed with the assumption that all authors have approved the submitted manuscript and concur in its submission to Visual Neuroscience. A Transfer of Copyright Agreement must be executed before an article can be published. Government authors whose articles were created in the course of their employment must so certify in lieu of copyright transfer. Authors are responsible for obtaining written permission from the copyright owners to reprint any previously published material included in their article.

\section{MANUSCRIPT SUBMISSION AND REVIEW.}

\section{VISUAL NEUROSCIENCE ONLINE SUBMISSION}

All submissions to Visual Neuroscience should proceed online: http://mc.manuscriptcentral.com/cup/vns

For information on the new online submission and review system, please read the Tutorial for Authors or the Tutorial for Reviewers

http://mcv3help.manuscriptcentral.com/tutorials

For questions on the submission and reviewing process please contact the Editor or Associate Editors

\section{Editor}

Benjamin E. Reese

Neuroscience Research Institute, University of California

Santa Barbara, CA 93106-5060, USA

Email: vns@lifesci.ucsb.edu

\section{Associate Editors}

Lynne Kiorpes

Center for Neural Science, New York University

New York, NY 10003 USA

E-mail: lynne@cns.nyu.edu

Peter D. Lukasiewicz

Department of Ophthalmology

Washington University School of Medicine

Saint Lous, MO 63110 USA

E-mail: lukasiewicz@vision.wustl.edu

\section{Paul R. Martin}

National Vision Research Institute and

Department of Optometry \& Vision Sciences

University of Melbourne

Carlton, VIC 3053 Australia

E-mail: prmartin@unimelb.edu.au

David S. Williams

Jules Stein Eye Institute, UCLA School of Medicine

Los Angeles, CA 90095 USA

E-mail: dswilliams@ucla.edu

Subsequent correspondence should refer to the Manuscript Reference Number, which will appear on the Acknowledgment sent to the corresponding author. Each manuscript will normally be reviewed by at least two referees with relevant scientific experience. Authors may suggest appropriate reviewers, but final selection of referees will be made by the Editor. Reviewers are asked to evaluate manuscripts for their scientific merit and clarity of presentation and to voice any concerns related to the welfare of animal and human subjects. Every effort will be made to notify authors of the reviewers' recommendations within 4 weeks of receipt of a manuscript.

MANUSCRIPT LENGTH AND EXCESS PAGE CHARGES. Concisely written papers are more likely to receive favorable review than those judged to be excessively long, but page charges are not levied for any articles. Manuscripts submitted as Brief Communications should normally occupy no more than 4 printed pages, figures included (approximately 13 manuscript pages).

MANUSCRIPT PREPARATION AND STYLE. Manuscripts must be in English and typed double-spaced. Allow margins of about 1 " $(20 \mathrm{~mm})$, using a 5 -space paragraph indent. Do not hyphenate words at the end of lines and do not justify right margins. Numbers should be spelled out when they occur at the

eginning of a sentence; use Arabic numerals elsewhere. Abbreviations should be used sparingly and nonstandard abbreviations should be defined at their first occurrence. Metric system (SI) units should be used. Manuscripts that do not conform to the style of Visual Neuroscience may be returned to the corresponding author without review. Authors of accepted manuscripts will be required to follow the instructions to authors for Online Submission.

MANUSCRIPT ELEMENTS AND ORDER. Unless there are obvious and compelling reasons for variation (e.g. Review Articles, Brief Communications), manuscripts should be organized as follows:

Title page. The title should be concise, informative, and free of abbreviations, chemical formulae, technical jargon, and esoteric terms. This page should include (a) the full title of the article, (b) names and affiliations of all authors, (c) the name, mailing address, telephone and fax numbers, and E-mail address for editorial correspondence, (d) the address for correspondence and/or reprint requests if different from the foregoing address, (e) a short title of 50 characters or less, and (f) a list of the number of manuscript pages, number of tables, and number of figures.

Abstract and keywords page. The second page of the manuscript should include (a) the article's full title, (b) an abstract of no more than 300 words, and (c) up to 5 keywords associated with the content and major thrust of the article. The abstract should give a succinct account of the objective, methods, results, and significance of the research

Introduction. This section begins on page 3 and should clearly state the objective of the research in the context of previous work bearing directly on the subject.

Materials and methods. This section should be brief but provide sufficient information to permit others to replicate the study. Pertinent details of species, apparatus and equipment, procedures and experimental design should be described.

Data from images. Methods of data acquisition, image processing, and figure preparation should be specified when images serve as the basis for quantitative data. This refers to optical, confocal, and CCD images as well as to images of gels, immunoblots, histological sections, etc.

All experiments involving human subjects must be conducted in accordance with principles embodied in the Declaration of Helsinki (Code of Ethics of the World Medical Association). Experiments involving animal subjects must conform to the principles regarding the care and use of animals adopted by the American Physiological Society and the Society for Neuroscience. The editor may refuse papers that provide insufficient evidence of adherence to these principles.

Results. The results should be presented clearly and concisely, using figures and tables to summarize or illustrate the important findings. Quantitative observations are often more effectively displayed in graphs than in tables.

Discussion. The discussion should summarize the major findings and explain their significance in terms of the objectives of the study and relationship to previous work. This section should present compact, clearly developed arguments rather than wideranging speculation or uncritical collation of earlier reports.

Acknowledgments. Use a separate page to recognize the contributions of individuals and supporting institutions.

References. Visual Neuroscience uses the author-date reference style of the Journal of Physiology. In the text, references should be cited as follows: as shown by Herrick (1948)

(Gordon et al., 1973)

(Buhl \& Peichl, 1986; Gordon et al., 1987)

The alphabetical list of references begins a new page, and must be typed double-spaced. Each in-text citation must have orresponding reference and vice versa. List works by different authors who are cited within the same parentheses in chronoogical order, beginning with the earlier work. Journal titles hould not be abbreviated. Only published articles and articles in press should appear in this list. Responsibility for the accuacy of references cited lies with the authors. Brief examples:

\section{Journal article}

BuHL, E.H. \& PeICHL, L. (1986). Morphology of rabbit retinal ganglion cells projecting to the medial terminal nucleus of the accessory optic system. Journal of Comparative Neurology 253, 163-174.

\section{Book}

HerRick, C.J. (1948). The Brain of the Tiger Salamander Chicago: University of Chicago Press.

\section{Chapter in an edited book}

Bonds, A.B. \& DeBRUYN, E.J. (1986). Inhibition and spatial selectivity in the visual cortex: The cooperative neuronal network revisited. In Models of Visual Cortex, ed. Rose, D. \& Dobson, V.G., pp. 292-300. Chichester, England: John Wiley \& Sons.

For more than one work by the same author(s) published in the same year, use (Jones, 1986a,b) in text and likewise in the reference section.

Tables. Tables should be numbered consecutively with Arabic umerals and each should be typed double-spaced on a separate page. All tables are to be submitted as separate files. A short explanatory title and column headings should make the table ntelligible without reference to the text. All tables must be cited and their approximate positions indicated in the text.

Figures and legends. The number of figures should be the minimum necessary to make the essential points of the paper Figures should be composed to occupy a single column $(8.3 \mathrm{~cm})$ or two columns $(17 \mathrm{~cm})$. Diagrams and illustrations must have a professional appearance and be prepared to permit reduction. To assure legibility, letters, numbers, and symbols on figures should have a minimum height of $1 \mathrm{~mm}$ when reduced. Photomicrographs must include a calibration bar; if symbols are used on micrographs, they must contrast sufficiently with the background to be clearly visible when printed. Color figures may be published at no charge in the online edition; a charge of $\$ 400$ or the first color figure, and $\$ 250$ for subsequent color figures, will be levied for authors requesting color reproduction in the printed version of the journal. Each figure must be cited and its approximate position clearly indicated within the text. Figures must be numbered consecutively and be accompanied by a descriptive caption typed double-spaced on a separate page from the figure. The captions, collected at the end of the manuscript, should concisely describe the figure and identify any symbols and/or calibration bars. Each figure must be submitted electroncally as a separate file. Electronic versions of figures should be submitted as TIFF or EPS files at $100 \%$ of a suitable final size. Color images for print should be $300 \mathrm{dpi}$ and prepared in CMYK mode; halftone and grayscale figures should be $300 \mathrm{dpi}$; line artwork should be 1200 dpi. (Please note that each manuscript can have up to $200 \mathrm{MB}$ of uploaded files, and that the LZW compression option in Adobe Illustrator is acceptable as well.)

COPYEDITING AND PAGE PROOFS. The publisher reserves the right to copyedit manuscripts to conform to the style of Visual Neuroscience. The corresponding author will receive page proofs for final proofreading. No rewriting of the final accepted manuscript is permitted at the proof stage, and substantial changes may be charged to the authors.

OFFPRINTS. The corresponding author will receive 25 free article offprints. A form will accompany the page proofs allowing orders for complete copies of the issue and for the purchase of additional offprints. Offprint requirements of all coauthors should be included on this form. 


\section{visual neuroscience}

Volume 26

\begin{abstract}
Wilson S. Geisler AND DARio Ringach
Jeremy R. Manning and David H. Brainard

Jan Wiltschut And Fred H. HAMkeR
\end{abstract}

Thorsten Hansen and Karl R. Gegenfurtner

\author{
Michael F. LAND \\ Hal S. Greenwald and David C. Knill \\ Mary Hayhoe, Barbara Gillam, Kelly Chajka, \\ and Elia Vecellio
}

Constantin A. Rothkopf And Dana H. Ballard

Sheng Zhang, Craig K. Abbey, AND Miguel P. ECKSTEIN

Wilson S. Geisler and Jeffrey S. Perry

ANTONIO TORRALBA

Igor Juricevic and Michael A. Webster

Laurence T. Maloney and Pascal Mamassian

Jan Wiltschut AND Fred H. Hamker
January/February 2009

Number 1

\section{CONTENTS}

\section{Introduction}

$1 \quad$ Natural systems analysis

\section{Natural Scene Statistics and Efficient Coding}

5 Optimal design of photoreceptor mosaics: Why we do not see color at night

21 Efficient coding correlates with spatial frequency tuning in a model of V1 receptive field organization

Independence of color and luminance edges in natural scenes

\section{Natural Tasks}

51 Vision, eye movements, and natural behavior

63 A comparison of visuomotor cue integration strategies for object placement and prehension

73 The role of binocular vision in walking

\section{Natural Scene Statistics and Natural Tasks}

81 Image statistics at the point of gaze during human navigation

93 Virtual evolution for visual search in natural images results in behavioral receptive fields with inhibitory surrounds

109 Contour statistics in natural images: Grouping across occlusions

123 How many pixels make an image?

\section{Natural Tasks and Plasticity}

133 Variations in normal color vision. V. Simulations of adaptation to natural color environments

147 Bayesian decision theory as a model of human visual perception: Testing Bayesian transfer

\section{Corrigendum}

157
Efficient coding correlates with spatial frequency tuning in a model of V1 receptive field organization 